\title{
FINITELY GENERATED CODINGS AND THE DEGREES R.E. IN A DEGREE d
}

\author{
RICHARD A. SHORE ${ }^{1}$
}

\begin{abstract}
We introduce finitely generated (partial) lattices which can be used to code an arbitrary set $D$. Results of Lerman, Shore and Soare are used to embed these lattices in the degrees r.e. in $D$. Thus if the degrees r.e. in and above $d$ are isomorphic to those r.e. in and above $\mathrm{c}, \mathrm{d}$ and $\mathrm{c}$ are of the same arithmetic degree. Similar applications are given to generic degrees and general homogeneity questions.
\end{abstract}

After the fact one might say that the main purpose of this paper is to introduce some schemes for doing coding in degree theory via finitely generated sets of degrees as opposed to the usual methods that employ definable substructures. Indeed we will describe such schemes and a number of applications to problems in degree theory. Truthfully, however, the motivation for this paper was the conjecture of Sacks [1966, p. 171] that $\operatorname{RED}(a)$, the degrees recursively enumerable in and above $a$, are isomorphic to $\operatorname{RED}=\operatorname{RED}(0)$ for every degree a. Our main result will refute this conjecture. Indeed we will show that if $\operatorname{RED}(\mathbf{a}) \cong \operatorname{RED}(\mathbf{b})$ then $\mathbf{a}$ and $\mathbf{b}$ are contained in the same arithmetric degree.

As we have said that Sacks [1966] supplies the question behind this paper we should also note that Lerman, Shore and Soare [1981] will essentially supply the answer. In that paper we proved that the r.e. degrees are not $\boldsymbol{\aleph}_{0}$-categorical by embedding distinct partial lattices $\mathscr{P}_{n}$ in RED, all of which were generated (under $\wedge$ and $\vee$ ) by three elements. We will here use the natural limit $\mathcal{P}_{\omega}$ of these structures as the core of our coding scheme. Before describing $\mathscr{P}_{\omega}$ we restate the definition of a partial lattice.

Definition 1. A structure $\mathscr{P}=\langle P, \leqslant, \$, \vee, \wedge\rangle$ is a partial lattice if there is a partial order on $P$ containing $\leqslant$ and disjoint from such that

(1.1) $\forall a, b, c \in P(a \vee b=c \rightarrow c$ is the least upper bound of $a$ and $b$ in $P$ ),

(1.2) $\forall a, b, c \in P(a \wedge b=c \rightarrow c$ is the greatest lower bound of $a$ and $b$ in $P)$.

(Note that we are treating $\wedge$ and $\vee$ as partial functions.)

Now $\mathscr{P}_{\omega}$ has three generators $t_{0}^{0}, t_{1}^{0}$ and $t_{2}^{0}$. Its elements are $t_{0}^{n}, t_{1}^{n}, t_{2}^{n}, b_{0}^{n}, b_{1}^{n}$ and $b_{\hat{2}}^{n}$ for $n \in N$. The defining relations determining the structure of $\mathscr{P}_{\omega}$ are as follows.

Received by the editors October 30, 1980 and, in revised form, March 10, 1981. Presented to the Society at the Special Session in Recursion Theory at the October 1980 meeting in Kenosha, Wisconsin. 1980 Mathematics Subject Classification. Primary 03D25, 03D30.

Key words and phrases. Recursively enumerable degrees, homogeneity problems, generic degrees.

${ }^{1}$ The preparation of this paper was partially supported by NSF grant MCS80-03016. We would also like to thank Carl Jockusch, Jr. for several helpful comments and suggestions. 
(2.1) $t_{i}^{n} \wedge t_{j}^{n}=b_{k}^{n}$ for $n>0,\{i, j, k\}=\{0,1,2\}$, and

(2.2) $t_{i}^{n}=t_{i}^{n-1} \vee b_{i}^{n-1}$ for $n>1, i<3$.

The ordering is the obvious one induced by these relations and the requirement that all the named elements be distinct. See Figure 1 for a picture of $\mathscr{P}_{\omega}$ in which solid lines indicate infimums and dashed ones supremums.
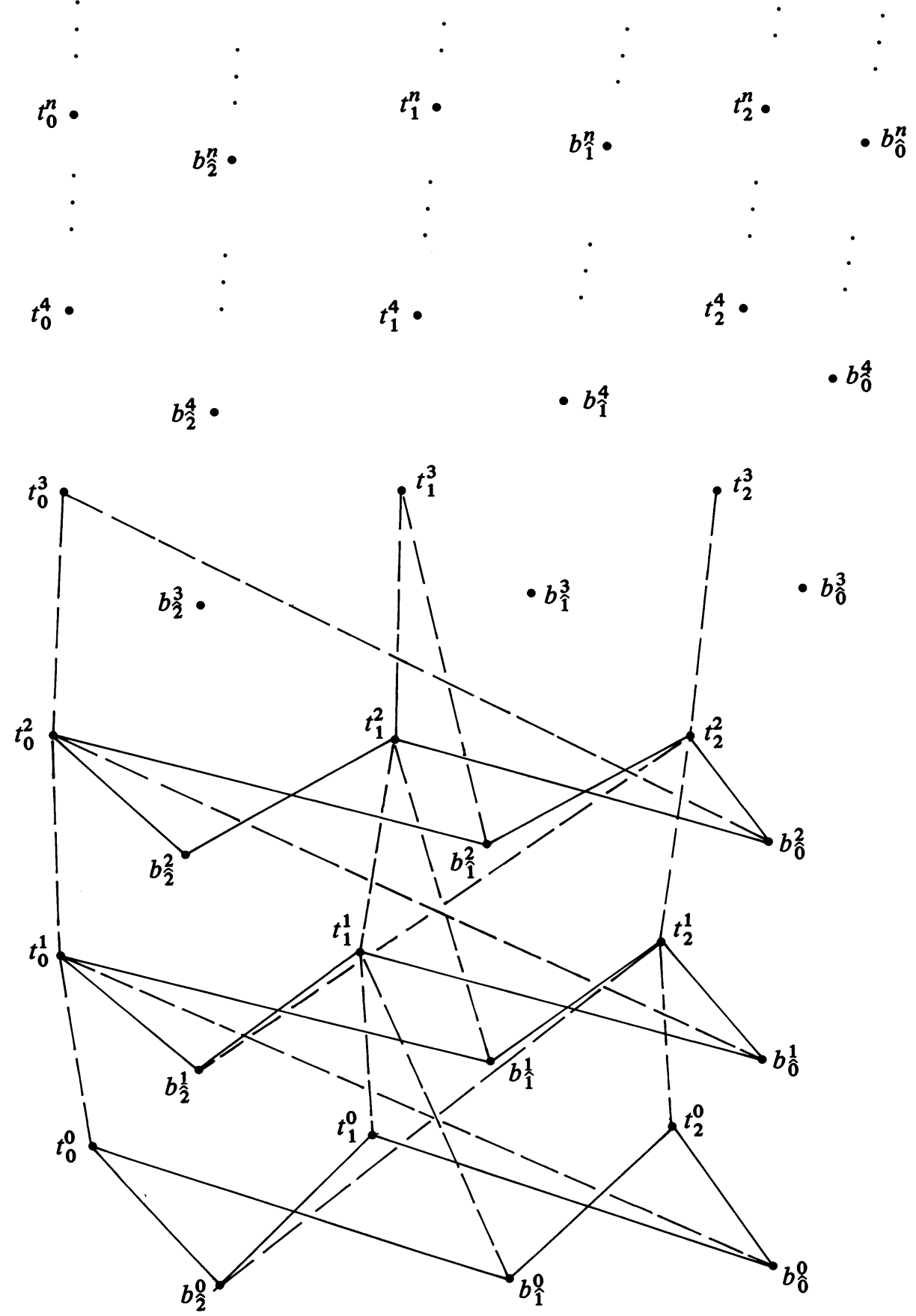

Figure 1 
We can now code an arbitrary set $\boldsymbol{A}$ into a finitely generated partial lattice $\mathscr{P}_{\boldsymbol{A}}$ by adding on one more generator $a$ incomparable with all the old elements, other new but not necessarily distinct elements $c_{n}$, and new relations given by

(2.3) $c_{n}=a \vee t_{2}^{n}$

(2.4) $n \in A \Leftrightarrow b_{2}^{n} \leqslant c_{n}$,

with all of the appropriate induced ordering relations and nonordering relations elsewhere. We claim that we can effectively recover the set $\boldsymbol{A}$ from the jump of any presentation of $\mathscr{P}_{A}$. Thus if $\mathscr{P}_{A}$ can be embedded in some upper semilattice $\mathcal{L}$, with $<, \$, \vee$ and $\wedge$ preserved, then $A$ is arithmetic in any presentation of $\mathcal{L}$. The point here is that we can start off with the elements representing $a, t_{0}^{0}, t_{1}^{0}$ and $t_{2}^{0}$. Assuming we have inductively calculated $t_{0}^{n}, t_{1}^{n}$ and $t_{2}^{n}$ it only takes a search recursive in the jump of the presentation of $\mathcal{L}$ even as a partial ordering to find the $b_{i}^{n}$ and $c_{n}$. We then ascertain if $n \in A$ by asking if $b_{2}^{n}<c_{n}$. To continue the induction we then find $t_{i}^{n+1}=t_{i}^{n} \vee b_{i}^{n}$ effectively in the presentation.

Now the proof of the main theorem of Lerman, Shore and Soare [1981] actually shows that any recursively presented partial lattice $\mathscr{P}$ having a property called TPP can be embedded in RED.

Definition 3. A partial lattice $\mathscr{P}$ has the trace probe property (TPP) if, for all $p, q \in P$ such that $p \$ q$, there is a finite sequence $Q_{0},\left\{q_{0}\right\}, Q_{1},\left\{q_{1}\right\}, \ldots, Q_{n}$ of subsets of $P$ such that

(3.1) $Q_{0} \subseteq Q_{1} \subseteq \cdots \subseteq Q_{n}$.

(3.2) $p \in Q_{0}$ and for all $r \in Q_{0} \cup\left\{q_{0}\right\}$ it is not the case that $r \leqslant q$.

(3.3) For all $i<n, Q_{i} \cup\left\{q_{i}\right\}$ and $\left\{q_{i}\right\}$ are trace complete as is $Q_{n}$. $(Q \subseteq P$ is trace complete if, for all $q \in Q$ and $a, b, c \in P$ with $q<c=a \vee b$, there is a $p \in Q$ such that $p \leqslant a$ or $p \leqslant b$.)

(3.4) For all $a, b, c \in P$ such that $a \wedge b=c$ and $i \leqslant n$, if there are $a_{1}, b_{1} \in Q_{i}$ such that $a_{1} \leqslant a$ and $b_{1} \leqslant b$, then either $i=0$ and there is an $r \in Q_{0}$ such that $r \leqslant c$, or $i>0$ and either there is an $r \in Q_{i-1}$ with $r \leqslant c$ or no element of $Q_{i-1} \cup\left\{q_{i-1}\right\}$ is $\leqslant a$ or no element of $Q_{i-1} \cup\left\{q_{i-1}\right\}$ is $\leqslant b$.

(3.5) For all $a, b, c \in P$ such that $a \wedge b=c$ and all $i<m$, either not $q_{i} \leqslant a$ or not $q_{i} \leqslant b$.

Relativizing the proof shows that if $\mathscr{P}$ is d-presentable and has TPP then it can be embedded in RED(d). As for the $\mathscr{P}_{n}$ it is a bit easier to use a modified version $\mathscr{P}_{A}^{*}$ of $\mathscr{P}_{A}$ when verifying TPP. $\mathscr{P}_{A}^{*}$ has the same elements as $\mathscr{P}_{A}$ and $\vee, \wedge$ and $<$ also are identical. We define $\$$ to hold in $\mathscr{P}_{A}^{*}$ only in the following cases:

(2.5) $b_{j}^{m} \nless t_{j}^{m}$ for $j \leqslant 2, m \in \omega$, and

(2.6) $b_{2}^{n} \nless c_{n}$ if $n \notin A$.

Now (2.5) clearly guarantees that the $t_{j}^{m}$ and $b_{j}^{m}$ are all distinct, and then (2.6) shows that the recovery of $\boldsymbol{A}$ from any presentation of any partial ordering extending $\mathscr{P}_{A}^{*}$ must be correct.

The verification that $\mathscr{P}_{A}^{*}$ has TPP is like that for the $\mathscr{P}_{n}^{*}$. We must define the required sequence $Q_{0},\left\{q_{0}\right\}, Q_{1},\left\{q_{1}\right\}, \ldots$, for each of (2.5) and (2.6). Suppose we are considering $b_{j}^{m}$ on the left of the $\$$ relation. Fix $i<k$ such that $\{i, j, k\}=$ $\{0,1,2\}$. We set $Q_{0}=\left\{b_{j}^{m}, t_{i}^{0}\right\}$ unless $b_{j}^{m} \leqslant c_{m}$ in which case we let $Q_{0}=$ $\left\{b_{j}^{m}, t_{i}^{0}, a\right\}$. In either case $q_{0}=t_{k}^{0}$. Suppose by induction $Q_{u}$ and $q_{u}$ have been 
defined and that $q_{u}=t_{v}^{0}$ for $v \in\{j, k\}$. Let $\left\{v, v^{*}\right\}=\{j, k\}$. Define $Q_{u+1}=Q_{u}$ $\cup\left\{b_{i}^{m-u-1}\right\}$ unless $b_{i}^{m-u-1}<c_{m-u-1}$ and $a \notin Q_{u}$. In this case let $Q_{u+1}=Q_{u} \cup$ $\left\{b_{v}^{m-u-1}, a\right\}$. In either case set $q_{u+1}=t_{v^{* *}}^{0}$ This procedure continues until $Q_{m}$ is defined and so $m=n$ in the definition of TPP. It is now straightforward to check that the clauses of TPP are satisfied and we omit this verification.

Thus for any degree a and set $A \in \mathbf{a}, \mathscr{P}_{A}^{*}$ is embeddable in $\operatorname{RED}(\mathbf{a})$. Now if $\operatorname{RED}(a) \cong \operatorname{RED}(b)$ then $\mathscr{P}_{A}^{*}$ can be embedded in $\operatorname{RED}(b)$ and so $A$ is recursive in the jump of any presentation of RED(b) as an upper semilattice. The standard presentation of RED(b) in terms of indices $W_{e}^{B}$ for some fixed $B \in \mathbf{b}$ is recursive in $B^{(4)}$ and so $A \leqslant B^{(5)}$. Similarly $B \leqslant A^{(5)}$.

THEOREM 4. If $R E D(a) \cong R E D(b)$ then $\mathbf{a}$ and $\mathrm{b}$ are contained in the same arithmetic degree. Indeed, $\mathbf{a} \leqslant \mathbf{b}^{(5)}$.

The calculation that gives $\mathbf{a} \leqslant \mathbf{b}^{(5)}$ here can be improved by embedding more complicated partial orderings in $\operatorname{RED(a).~It~is~immediate~from~the~proof~of~}$ Lerman, Shore and Soare [1981] and the limit lemma that any $a^{\prime}$-presentable partial lattice having TPP can be embedded in RED(a). A bit more care would show that $\mathscr{P}_{C}^{*}$ is embeddable in $\operatorname{RED}(a)$ for any $C$ which is $\Pi_{2}$ in $A$. Thus if $\operatorname{RED(a)\cong ~}$ $\operatorname{RED}(\mathbf{b})$ then $\mathbf{a}^{(2)} \leqslant \mathbf{b}^{(5)}$.

COROLlary 5. The theory of RED(a) with added parameters is not recursive in a.

Proof. Add on parameters corresponding to degrees $\mathbf{t}_{0}^{0}, \mathbf{t}_{1}^{0}, \mathbf{t}_{2}^{0}$ and $\mathbf{b}$ to generate $\mathscr{P}_{B}$ with $B \in \mathbf{a}^{\prime}$.

COROLlaRY 6. The structure $R E D(\mathbf{a})$ is not presentable recursively in a even as a partial ordering.

Proof. If it were and some elements $\mathbf{t}_{0}^{0}, \mathbf{t}_{1}^{0}, \mathbf{t}_{2}^{0}, \mathbf{c}$ in it generate a partial lattice $\mathscr{P}_{C}$, then $C \leqslant \mathbf{a}^{\prime}$ as we have argued. But we can in $\operatorname{RED(a)}$ generate $\mathscr{P}_{C}$ with $C \in \mathbf{a}^{\prime \prime}$ for a contradiction.

Turning now to the degrees as a whole, we first note that the recoverability of $A$ from the jump of the presentation of any partial order in which $\mathscr{P}_{A}$ is embedded and the fact that one can easily extend $\mathscr{P}_{A}$ to a full lattice show that not every

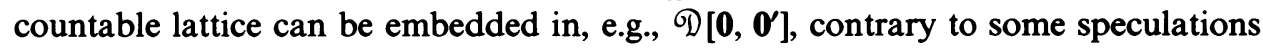
in Posner [1980, p. 59]. On the other hand, there are, as Posner there expects, methods of embedding simply presented lattices in $\mathscr{D}\left[0,0^{\prime}\right]$ much easier than the initial segments methods of Lerman [1982]. Indeed we can embed $\mathscr{P}_{A}$ or any a-presentable lattice in $\mathscr{D}\left[\mathbf{a}, \mathbf{a}^{\prime}\right]$ using finite extension methods in the style of Kleene and Post [1955]. One also needs a simple representation theorem.

THEOREM 7. Let $\left\{p_{i}\right\}$ enumerate an a-presentable partial lattice $\mathcal{P}$ (i.e. $<, \$, \vee$ and $\wedge$ are partial recursive in a relations) for which $<$ and $\$$ define a partial order on $\mathcal{P}$. There is then a uniformly recursive in a array of functions $\alpha_{n}$ such that

$$
\begin{gathered}
p_{i} \leqslant p_{j} \Leftrightarrow \forall n, m\left[\alpha_{n}(j)=\alpha_{m}(j) \rightarrow \alpha_{n}(i)=\alpha_{m}(i)\right], \\
p_{i} \vee p_{j}=p_{k} \Rightarrow \forall n, m\left[\alpha_{n}(i)=\alpha_{m}(i) \& \alpha_{n}(j)=\alpha_{m}(j) \rightarrow \alpha_{n}(k)=\alpha_{m}(k)\right],
\end{gathered}
$$




$$
\begin{aligned}
& p_{i} \wedge p_{j}=p_{k} \& \alpha_{n}(k)=\alpha_{m}(k) \Rightarrow \\
& \quad \exists q_{1}, q_{2}, q_{3}\left[\alpha_{n}(i)=\alpha_{q_{1}}(i) \& \alpha_{q_{1}}(j)=\alpha_{q_{2}}(j) \& \alpha_{q_{2}}(i)=\alpha_{q_{3}}(i) \& \alpha_{q_{3}}(j)=\alpha_{m}(j)\right] .
\end{aligned}
$$

Proof. This is essentially Jonsson [1953] translated into the language of Lerman [1971] or [1982] which we reproduce here. First we build a representation $\left\{\beta_{n}\right\}$ satisfying (7.1) and (7.2):

$$
\begin{aligned}
\beta_{2 s+1}(i) & =2^{2 s+1} \quad \forall i, \\
\beta_{2 s}(i) & = \begin{cases}2^{2 s+1} \quad \text { if } p_{i} \leqslant p_{s}, \\
2^{2 s} \text { if } p_{i} \nless p_{s} .\end{cases}
\end{aligned}
$$

The check that (7.1) and (7.2) hold is routine.

For any situation as in the hypothesis of (7.3) we can add on the required three functions and still preserve (7.1) and (7.2):

Suppose $p_{i} \wedge p_{j}=p_{k}$ and $\gamma(k)=\delta(k)$. If $p_{i} \leqslant p_{j}$ then we can set $q_{1}=q_{2}=q_{3}=$ $\delta$. Otherwise we let $w, x, y$ and $z$ be new numbers not in the range of any of our functions. Now let

$$
\begin{aligned}
& q_{1}(n)= \begin{cases}\gamma(n) & \text { if } p_{n} \leqslant p_{i}, \\
w & \text { if } p_{n} \leqslant p_{i} ;\end{cases} \\
& q_{2}(n)= \begin{cases}\gamma(n) & \text { if } p_{n} \leqslant p_{j}, \\
x & \text { if } p_{n} \leqslant p_{i} \& p_{n} \leqslant p_{j}, \\
y & \text { otherwise; }\end{cases} \\
& q_{3}(n)= \begin{cases}\delta(n) & \text { if } p_{n} \leqslant p_{j}, \\
x & \text { if } p_{n} \leqslant p_{i} \& p_{n} \\
z & \text { otherwise. }\end{cases}
\end{aligned}
$$

Again a straightforward check shows that (7.3) is now satisfied for this situation and (7.1) and (7.2) remain valid. We can therefore close off under this process to generate effectively in the presentation of $\mathscr{P}$ a representation with all the required properties.

Now suppose we are given an a-presentable partial lattice $\mathscr{P}=\left\{p_{i}\right\}$ that we wish to embed in $\mathscr{D}\left[\mathbf{a}, \mathbf{a}^{\prime}\right]$. We assume that $\leqslant$ and give a complete partial order on $\mathcal{P}$ and so choose a representation $\left\{\alpha_{n}\right\}$ as in Theorem 7 . We will build a function $g \leqslant \mathbf{a}^{\prime}$ by specifying finite initial segments of $g$ in a construction recursive in $\mathbf{a}^{\prime}$. Our embedding $\mathscr{P} \rightarrow \mathscr{D}\left[\mathbf{a}, \mathbf{a}^{\prime}\right]$ will then be given by $p_{i} \mapsto a \vee h_{i}$ where $a \in \mathbf{a}$ and $h_{i}(n)=\alpha_{q(n)}(i)$. [If we wish to make the dependence of $h_{i}$ on $g$ explicit we write it as $h_{i}(g)$.] As $g \leqslant \mathbf{a}^{\prime}, \operatorname{deg}\left(a \vee h_{i}\right) \in \mathscr{D}\left[\mathbf{a}, \mathbf{a}^{\prime}\right]$. Moreover if $p_{i} \leqslant p_{j}$ then $h_{i} \leqslant{ }_{T} a \vee h_{j}$, for to compute $h_{i}(n)=\alpha_{g(n)}(i)$ we first compute $h_{j}(n)=\alpha_{g(n)}(j)$. We then find any $m$ such that $\alpha_{m}(j)=h_{j}(n)$. By (7.1) $\alpha_{m}(i)=\alpha_{g(n)}(i)=h_{i}(n)$. We next note that if $p_{i} \vee p_{j}=p_{k}$ then $h_{k} \leqslant{ }_{T} a \bigvee h_{i} \vee h_{j}$. To calculate $h_{k}(n)$ compute $h_{i}(n)=\alpha_{g(n)}(i)$ and $h_{j}(n)=\alpha_{g(n)}(j)$. Then find any $m$ such that $\alpha_{m}(i)=h_{i}(m)$ and $\alpha_{m}(j)=h_{j}(n)$. By (7.2) $\alpha_{m}(k)=\alpha_{g(n)}(k)=h_{k}(n)$. Thus our embedding of $\mathcal{P}$ into $\mathscr{D}\left[\mathbf{a}, \mathbf{a}^{\prime}\right]$ will preserve $\leqslant$ and $\vee$. We must take steps in the construction of $g$ to preserve $\$$ and $\wedge$ as well. We will write $h_{i}$ for $a \vee h_{i}$ below. 
Stage $s=\langle 0, e, i, j\rangle$. Suppose we have defined $g^{s}$ with dom $g^{s}=t$. If $p_{i} \leqslant p_{j}$, we go to the next stage. Otherwise we let $n$ and $m$ witness that $p_{i} \nless p_{j}$ as in (7.1). Ask (of $a^{\prime}$ ) if there is any finite extension $g^{\prime}$ of $g^{s}$ such that $g^{\prime}(t)=n$ and $\{e\}^{h\left(g^{\prime}\right)}(t)$ is convergent. If not let $g^{s+1}=g^{s} \cup\{\langle t, n\rangle\}$ and go on. If so we may choose such a $g^{\prime}$ with $\{e\}^{h_{j}\left(g^{\prime}\right)}(t) \neq \alpha_{g^{\prime}(t)}(i)$ by switching the value of $g^{\prime}(t)$ from $n$ to $m$ if necessary since this changes $\alpha_{g^{\prime}(t)}(i)$ but does not affect $h_{j}\left(g^{\prime}\right)$ by our choice of $n$ and $m$. We then let $g^{s+1}=g^{\prime}$ and proceed.

Stage $s=\langle u, e, i, j\rangle, u\rangle 0$. If $p_{i} \wedge p_{j}=p_{k}$ is not verified in $u$ many steps, we go on. If it is, we ask (of $a^{\prime}$ ) if there are finite extensions $g_{1}$ and $g_{2}$ of $g^{s}$ with dom $g_{1}=\operatorname{dom} g_{2}$ and

$$
\alpha_{g_{1}(n)}(k)=\alpha_{g_{2}(n)}(k) \quad \forall n \in \operatorname{dom} g_{1},
$$

and an $x$ such that

$$
\{e\}^{h_{i}\left(g_{1}\right)}(x) \neq\{e\}^{h_{j}\left(g_{2}\right)}(x)
$$

If not we let $g^{s+1}=g^{s}$ and go on. If so we let the finite functions $q_{1}, q_{2}$ and $q_{3}$ extending $g^{s}$ be as required by (7.3), i.e.

$$
\begin{aligned}
& \alpha_{g_{1}(n)}(i)=\alpha_{q_{1}(n)}(i), \quad \alpha_{q_{1}(n)}(j)=\alpha_{q_{2}(n)}(j), \\
& \alpha_{q_{2}(n)}(i)=\alpha_{q_{3}(n)}(i) \quad \text { and } \quad \alpha_{q_{3}(n)}(j)=\alpha_{g_{2}(n)}(j)
\end{aligned}
$$

for every $n \in \operatorname{dom} g_{1}$. (We naturally get $\operatorname{dom} q_{1}=\operatorname{dom} q_{2}=\operatorname{dom} q_{3}=\operatorname{dom} g_{1}$.) Thus $\{e\}^{h_{i}\left(g_{1}\right)}(x)=\{e\}^{h_{i}\left(q_{1}\right)}(x)$ and $\{e\}^{h_{i}\left(g_{2}\right)}(x)=\{e\}^{h_{3}\left(q_{3}\right)}(x)$.

We now ask if $\exists q_{1}^{\prime}$ extending $q_{1}$ such that $\{e\}^{h_{(}\left(q_{1}^{\prime}\right)}(x) \downarrow$. If not let $g^{s+1}=q_{1}$ and go on. If so choose one. If $\{e\}^{h_{i}\left(q_{1}^{\prime}\right)}(x) \neq\{e\}^{h_{3}\left(q_{1}^{\prime}\right)}(x)$ let $g^{s+1}=q_{1}^{\prime}$. If not we have $\{e\}^{h_{i}\left(g_{1}\right)}(x)=\{e\}^{h_{i}\left(q_{1}\right)}(x)=\{e\}^{h_{i}\left(q_{1}^{\prime}\right)}(x)=\{e\}^{h_{j}\left(q_{1}^{\prime}\right)}(x)=\{e\}^{h_{j}\left(q_{2}^{\prime}\right)}(x)$ where $q_{2}^{\prime}$ is the extension of $q_{2}$ by the same string added on to $q_{1}$ to get $q_{1}^{\prime}$, so that $h_{j}\left(q_{1}^{\prime}\right)=h_{j}\left(q_{2}^{\prime}\right)$ by our choice of $q_{1}$ and $q_{2}$. We now ask if there is an extension $q_{2}^{\prime \prime}$ of $q_{2}^{\prime}$ such that $\{e\}^{h_{i}\left(q_{2}^{\prime \prime}\right)}(x) \downarrow$. If not we let $g^{s+1}=q_{2}^{\prime}$. If so we choose one. Again if $\{e\}^{h_{i}\left(q_{2}^{\prime \prime}\right)}(x) \neq$ $\{e\}^{h_{(}\left(q_{2}^{\prime \prime}\right)}(x)$ we let $g^{s+1}=q_{2}^{\prime \prime}$. Otherwise we have $\{e\}^{h_{1}\left(g_{1}\right)}(x)=\{e\}^{h_{1}\left(q_{2}^{\prime}\right)}(x)=$ $\{e\}^{h_{3}\left(q_{2}^{\prime \prime}\right)}(x)=\{e\}^{h_{i}\left(q_{2}^{\prime \prime}\right)}(x)=\{e\}^{h_{i}\left(q_{3}^{\prime \prime}\right)}(x)$ where $q_{3}^{\prime \prime}$ is the extension of $q_{3}$ gotten by adding on the same string that produces $q_{2}^{\prime \prime}$ from $q_{2}$ so that $h_{i}\left(q_{2}^{\prime \prime}\right)=h_{i}\left(q_{3}^{\prime \prime}\right)$ by our choice of $q_{2}$ and $q_{3}$. We now have $\{e\}^{h_{i}\left(q_{3}^{\prime \prime}\right)}(x)=\{e\}^{h_{i}\left(g_{1}\right)}(x) \neq\{e\}^{h_{1}\left(g_{2}\right)}(x)=$ $\{e\}^{h_{3}\left(q_{3}\right)}(x)=\{e\}^{h_{3}\left(q_{3}^{\prime \prime}\right)}(x)$ and so we let $g^{s+1}=q_{3}^{\prime \prime}$ and proceed.

Our construction guarantees that $\$$ and $\Lambda$ are preserved by the usual arguments. Thus we have proved the following.

THEOREM 8. Any a-recursively presented partial lattice which is a partial ordering such as $\mathscr{P}_{A}$ can be embedded in $\mathscr{D}\left[\mathbf{a}, \mathbf{a}^{\prime}\right]$.

One could of course improve on this result to embed more complicated lattices in $\mathscr{D}\left[\mathbf{a}, \mathbf{a}^{\prime}\right]$, but exactly which lattices are so embeddable is not clear. However this relatively simple result does imply that $A$ is recursive in the jump of any presentation of $\mathscr{D}\left[\mathbf{a}, \mathbf{a}^{\prime}\right]$. Thus it can be used to replace coding by the much more difficult initial segment techniques in various arguments. For example, it immediately refutes the strong homogeneity conjecture by showing that if $\mathscr{D}\left[\mathbf{a}, \mathbf{a}^{\prime}\right]=\mathscr{D}\left[\mathbf{b}, \mathbf{b}^{\prime}\right]$ 
then $\mathbf{a} \leqslant \mathbf{b}^{(5)}$ as $\mathscr{D}\left[\mathbf{b}, \mathbf{b}^{\prime}\right]$ is $\mathbf{b}^{(4)}$-presentable. Moreover, combined with the lemma of Harrington and Shore [1981] showing that $e^{a}=\{x>a \mid \exists y>x \forall z(z \vee y$ is not a minimal cover of $\mathbf{z}$ ) $\}$ is caught between $\mathcal{Q}^{\mathbf{a}}$ and $\mathcal{H C}^{\mathbf{a}}$ (the degrees above $\mathbf{a}$ of sets arithmetic and hyperarithmetic in a respectively), it refutes the homogeneity conjecture. Indeed it shows that if $\mathscr{D}(\geqslant \mathbf{a}) \cong \mathscr{D}(\geqslant \mathbf{b})$ then $\mathbf{a}$ and $\mathbf{b}$ are contained in the same hyperdegree.

For our final application of these codings, we note that by a variation on the usual permitting (or full approximation) methods the embedding of $\mathscr{P}_{A}$ into $\mathscr{D}\left[\mathbf{a}, \mathbf{a}^{\prime}\right]$ described above can be transformed to one into $\mathscr{D}[\mathbf{a}, \mathbf{c}]$ for any $\mathbf{c}>\mathbf{a}$ which is r.e. in $\mathbf{a}$.

The main problem is how to treat the multistep process used in the oracle construction at stages $s=\langle u, e, i, j\rangle$ with $u>0$ when $p_{i} \wedge p_{j}=p_{k}$. The idea is to run several searches and permitting waits in a row. At an appropriate spot $a$ (and all large enough stages $s$ ) we will first look for possible extensions $g_{1}$ and $g_{2}$ of $g^{s} \uparrow a$ as described above. If we find them we can also immediately find $q_{1}, q_{2}$ and $q_{3}$ as required. We then will switch to $q_{1}$ when we are permitted to do so. If we are so permitted we begin searching for $q_{1}^{\prime}$ as described above. If we find it and are once again permitted, we switch to $q_{1}^{\prime}$ if it produces the desired disagreement (thereby assuring success) or to $q_{2}^{\prime}$ if it does not. In this last case, we start our final search for $q_{2}^{\prime \prime}$. If we find it and are again permitted, we switch to $q_{2}^{\prime \prime}$ if it produces the desired disagreement and otherwise to $q_{3}^{\prime \prime}$.

The start-up procedures at new points are much as usual if one thinks of each subsearch as making progress. If at point $a$ we are looking for a certain extension but have not yet found it, no further activity is needed for this requirement at latter points. When we find it we start looking at some latter point $b$ for such extensions until we are permitted at $a$. In this construction we may be permitted at $a$ to make some progress which, rather than assuring success, requires another search. In this case we suspend work at $b$ until this next extension is found for $a$ (but is not yet permitted). One argues for the ultimate success of the construction by considering (after higher priority requirements have been satisfied) the last step in this process that we reach infinitely of ten but are never able to move beyond.

Consider now any 1-generic degrees $c_{1}, c_{2}$ as defined in Jockusch [1980]. By Theorem 5.1 of that paper $\mathbf{c}_{i}$ is r.e. in some $\mathbf{a}_{i}<\mathbf{c}_{i}$. Now $\mathscr{P}_{A_{i}}$ is embeddable in $\mathscr{D}\left[a_{i}, \mathbf{c}_{i}\right]$ and so in $\mathscr{D}\left(\leqslant \mathbf{c}_{i}\right)$. Thus if $\mathscr{D}\left(\leqslant c_{1}\right) \cong \mathscr{D}\left(\leqslant c_{2}\right)$ then $\mathbf{a}_{i} \leqslant \mathbf{c}_{j}^{(4)}$ as $\mathscr{D}\left(<\mathbf{c}_{i}\right)$ is $\mathbf{c}_{i}^{(3)}$-presentable. Of course $\mathbf{c}_{i} \leqslant \mathbf{a}_{i}^{\prime}$ and so we have the following.

THEOREM 9. If $\mathbf{c}_{1}$ and $\mathbf{c}_{2}$ are 1-generic degrees (or even each one r.e. in some degree below it) and $\mathscr{D}\left(\leqslant \mathrm{c}_{1}\right) \cong \mathscr{D}\left(\leqslant \mathrm{c}_{2}\right)$ then they are contained in the same arithmetic degree. Indeed $\mathbf{c}_{1} \leqslant \mathbf{c}_{2}^{(5)}$.

As there are continuum many generic degrees we immediately get

COROLlary 10. There are generic $\mathbf{c}_{1}$ and $\mathbf{c}_{2}$ with $\mathscr{D}\left(<c_{1}\right) \supsetneqq \mathscr{D}\left(\leqslant \mathbf{c}_{2}\right)$.

This answers Question 8 of Jockusch [1980]. 


\section{BIBLIOGRAPHY}

L. Harrington and R. A. Shore, [1981]. Definable degrees and automorphisms of $\mathscr{D}$, Bull. Amer. Math. Soc. (N.S.) 4, 97-100.

C. G. Jockusch, Jr., [1980]. Degrees of generic sets, Recursion Theory: Its Generalizations and Applications (F. R. Drake and S. S. Wainer, eds.), London Math. Soc. Lecture Notes, no. 45, Cambridge Univ. Press, pp. 110-139.

B. Jonsson, [1953]. On the representation of lattices, Math. Scand. 1, 193-206.

S. C. Kleene and E. L. Post, [1954]. The upper semi-lattice of degrees of unsolvability, Ann. of Math. (2) 59, 379-407.

M. Lerman, [1971]. Initial segments of the degrees of unsolvability, Ann. of Math. (2) 93, 365-389. , [1982]. Structure theory for the degrees of unsolvability, Springer-Verlag, Berlin (to appear).

M. Lerman, R. A. Shore and R. I. Soare, [1981]. The recursively emumerable degrees are not $N_{0}$-categorical, Adv. in Math. (to appear).

D. Posner, [1980]. A survey of the non- $R E$ degrees $<\boldsymbol{\sigma}$, Recursion Theory: Its Generalizations and Applications (F. R. Drake and S. S. Wainer, eds.), London Math. Soc. Lecture Notes, no. 45, Cambridge Univ. Press, pp. 52-109.

G. E. Sacks, [1966]. Degrees of unsolvability, 2nd ed., Ann. of Math. Studies, no. 55, Princeton Univ. Press, Princeton, N.J.

Department of Mathematics, Cornell University, Ithaca, New York 14853 